variant genotype for Apal and Taql was associated with a higher risk of fibromyalgia in SLE patients.

REFERENCES:

[1] Zhou TB, Jiang ZP, Lin ZJ, Su N. Association of vitamin D receptor gene polymorphism with the risk of systemic lupus erythematosus. J Recept Signal Transduct Res. 2015;35(1):8-14.

Acknowledgements: The Faculty of Medicine and Surgery, University of Malta provided funding for this research.

Disclosure of Interests: None declared

DOI: 10.1136/annrheumdis-2021-eular.770

\section{AB0078 LIPID IMMOBILIZATION AS A METHOD TO OBTAIN ANTIGENIC NANO-OBJECTS}

O. Rusanova ${ }^{1}$, O. Emelyanova ${ }^{1,2}$, N. Emelyanov ${ }^{2} .{ }^{1}$ Federal State Budgetary Institution «Research Institute of Clinical and Experimental Rheumatology named after A.B. Zborovsky», 1, Волгоград, Russian Federation; ${ }^{2}$ Federal State Budgetary Educational Institution of Higher Education «Volgoorad State Medical University» of the Ministry of Healthcare of the Russian Federation, 2, Волгоград, Russian Federation

Objectives: Objective of the study is to research the effect of emulsion polymerization on active sites of cardiolipin antigen determinant in antiphospholipid syndrome (APS) in patients with systemic lupus erythematosus (SLE).

Methods: Having integrated antigen nanoobjects we developed immobilized magnetocontrollable antigen nanosystems and put them to an evaluation test. The nanosystems are polyacrylamide granules with a built in antigen. To obtain stable immobilized multi-use biopharmaceuticals with targeted properties (shape, particle diameter, pore size, density) we used a modified version of emulsion polymerization method using polyacrylamide carrier gel. This method permitted a greater sorptive capacity, preserving the antigen in maximum native state, and opened up the possibility of controllable modification of nanoobjects. Cardiolipin was used as the antigen in question.

Results: Following the method described above we performed sorption of anticardiolipin antibodies from blood plasma of SLE patients who showed clinical presentations of antiphospholipid syndrome. Blood serum from 10 apparently healthy individuals served as control. The level of cardiolipin antibodies was determined before and after sorption by indirect solid phase immunoenzyme method. In the eluate we estimated total protein by Lowry method. In vitro testing showed that the obtained antigen nanosystems based on immobilized cardiolipin could effectively remove cardiolipin antibodies from whole blood of SLE patients with clinical presentations of APS to achieve the values of healthy individuals (before sorption cardiolipin antibodies $0.328 \pm 0.028$; after sorption $0.059 \pm$ $0.017 ; p<0.001$; sorption capacity $8.00 \pm 0.390 \mathrm{mg} / \mathrm{ml}$ ).

Conclusion: The method of emulsion polymerization with consideration to hydrophobic and hydrophilic properties of lipid molecules permits obtaining and modifying biomolecules with certain properties, in a controlled fashion.

Disclosure of Interests: None declared

DOI: 10.1136/annrheumdis-2021-eular.1075

\section{AB0079 ENDOTHELIAL FUNCTION IN SYSTEMIC LUPUS ERYTHEMATOSUS PATIENTS: IMPACT OF CAFFEINE CONSUMPTION ON ENDOTHELIAL PROGENITOR CELLS}

V. Orefice ${ }^{1}$, F. Ceccarelli ${ }^{1}$, C. Barbati ${ }^{1}$, E. Putro ${ }^{1}$, C. Pirone ${ }^{1}$, F. R. Spinelli ${ }^{1}$, C. Alessandri', F. Conti'. 'Lupus Clinic, Sapienza University of Rome, Department of Clinical Internal, Anesthesiological and Cardiovascular Sciences, Rome, Italy

Background: As widely demonstrated, circulating endothelial progenitor cells (EPCs) could be considered biomarkers of endothelial dysfunction. Their frequency and function varied in systemic lupus erythematosus (SLE) patients, with a significant association with subclinical atherosclerosis ${ }^{1}$. Caffeine, one of the most widely consumed products in the world, seems to interact with multiple components of the immune system by acting as a non-specific phosphodiesterase inhibitor $^{2}$. In terms of cardiovascular disease (CVD), data from the literature showed a U-shaped association between habitual coffee intake and $C V D^{3}$. In this view, Spyridopoulos et al. demonstrated a significant improvement in mature endothelial cells and EPCs migration in relation to coffee consumption in coronary artery disease both in mouse models and in patients ${ }^{4}$. Finally, caffeine seems to play a positive effect on SLE disease activity status, as demonstrated by the inverse association between its intake and SLE Disease Activity Index 2000 (SLEDAl-2K) and the serum levels of inflammatory cytokines ${ }^{5}$. At the best of our knowledge, there are no data about the effect of caffeine on cardiovascular risk in SLE patients. Objectives: The aim of this study was to evaluate the possible role of caffeine intake on endothelial function in SLE patients, by evaluating its effect on circulating EPCs.
Methods: We performed a cross-sectional study enrolling SLE patients, fulfilling the revised 1997 ACR criteria. According with the protocol study, we excluded patients with history of smoking, CVD, chronic kidney failure, dyslipidaemia, and/or diabetes. At recruitment, the clinical and laboratory data were collected and disease activity was assessed using the SLEDAl-2k. Caffeine intake was evaluated using a 7-day food frequency questionnaire, previously employed in SLE cohort ${ }^{5}$. At the end of questionnaire filling, blood samples were collected. EPCs were isolated from peripheral blood mononuclear cells (PBMC) by a flow cytometry analysis and they were defined as early EPCs CD34+KDR+CD133+ cells and late EPCs CD34+KDR+CD133-, expressed as a percentage within the lymphocyte gate.

Results: We enrolled 19 patients (F:M 18:1, median age 45 years, IQR 15 median disease duration 240 months, IQR 168). In this cohort, we observed a mean \pm SD SLEDAI- $2 \mathrm{k}$ value of $1.3 \pm 3.3$ and the most frequent disease-related feature was joint involvement $(73.7 \%)$. Concerning treatment at the time of enrolment, the majority of patients were receiving treatment with hydroxychloroquine $(78.9 \%)$ and seven with glucocorticoids $(36.8 \%)$. The median intake of caffeine was $163 \mathrm{mg} /$ day (IQR 138) and we used this value as cut-off to categorize SLE patients in 2 groups: group $1(\mathrm{~N}=10$, caffeine intake $\leq 163 \mathrm{mg} /$ day) and group 2 $(\mathrm{N}=9$, caffeine intake $>163 \mathrm{mg} /$ day). Patients with less intake of caffeine showed a significantly more frequent history of lupus nephritis $(p=0.03)$, haematological manifestations $(p=0.0003)$ and anti-dsDNA positivity $(p=0.0003)$. Moving on EPCs, a positive correlation between caffeine intake and EPCs percentage was observed $(p=0.04, r=0.4)$ (Figure $1 A)$. Moreover, patients with more caffeine intake showed higher levels of early EPCs ( $p=0.02)$ (Figure 1B)

Conclusion: This is the first report analysing the impact of caffeine on EPCs frequency in SLE patients. We found a positive correlation between its intake and both early and late EPCs percentage, suggesting a caffeine influence on endothelial function in SLE patients. Nonetheless, these results support the possible impact of dietary habits on autoimmune diseases.

\section{REFERENCES:}

[1] Westerweel et al. Ann Rheum Dis 2007.

[2] Aronsen et al. Europ Joul of Pharm 2014.

[3] Ding et al. Circulation 2015

[4] Spyridopoulos et al. Art. Thromb Vasc Biol. 2008.

[5] Orefice et al. Lupus 2020.

Figure 1. A. Correlation between $K D R+C D 34+$ cells and daily caffeine intake B. $K D R+C D 34+C D 133+$ cells percentage in group $1(\mathrm{~N}=10$, caffeine intake $\leq 163 \mathrm{mg} /$ day) and group 2 $(\mathrm{N}=9$, caffeine intake $>163 \mathrm{mg} / \mathrm{day})$
A

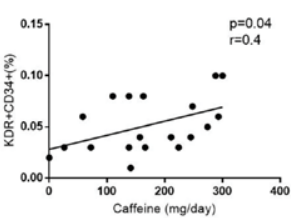

B

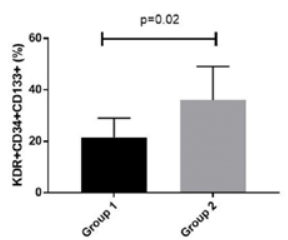

Disclosure of Interests: None declared

DOI: 10.1136/annrheumdis-2021-eular.1224

\section{\begin{tabular}{|l|l}
\hline AB0080 & ALTERED CONCENTRATIONS OF DIFFERENT
\end{tabular} SMALL EXTRACELLULAR VESICLE POPULATIONS IN PLASMA OF PATIENTS WITH ANTIPHOSPHOLIPID SYNDROME}

U. Stok $^{1,2}$, A. Shephard ${ }^{3}$, S. Cucnik ${ }^{1,2}$, S. Sodin-Šemrl ${ }^{1,4}$, P. Zigon ${ }^{1,4}{ }^{1}{ }^{1}$ University Medical Centre Ljubljana, Department of Rheumatology, Ljubljana, Slovenia; ${ }^{2}$ University of Ljubljana, Faculty of Pharmacy, Ljubljana, Slovenia; ${ }^{3}$ Nanoview Biosciences, Research and Development, Malvern, United Kingdom; ${ }^{4}$ University of Primorska, Faculty of Mathematics, Natural Sciences and Information Technologies, Koper, Slovenia

Background: Antiphospholipid syndrome (APS) is a systemic autoimmune disorder characterized by thrombosis, obstetric complications, and the presence of antiphospholipid antibodies (aPL) that cause endothelial injury and thrombophilia [1]. Extracellular vesicles (EVs) are involved in various thrombotic disorders [2], including APS [3,4], and therefore may influence the prothrombotic status of APS patients. One of the hallmarks of activated endothelium is the expression of adhesion molecules, such as ICAM-1 (CD54) and E-selectin (CD62E), that play a key function in the interactions with leukocytes and platelets.

Objectives: To determine the level of total tetraspanin (CD81/CD63/CD9)-positive vesicles and specific EV populations (CD54- and CD62E-positive EVs) in plasma from APS patients.

Methods: Whole blood was collected from 4 APS patients and 3 healthy blood donors (HBDs) and processed to obtain platelet-depleted plasma. The size and concentration of EVs were determined using ExoView platform (NanoView 